\title{
Reactivation of Rate Remapping in CA3
}

\author{
C. Daniela Schwindel, ${ }^{1}$ Zaneta Navratilova, ${ }^{1}$ Karim Ali, ${ }^{1}$ Masami Tatsuno, ${ }^{1}$ and Bruce L. McNaughton ${ }^{1,2,3}$ \\ ${ }^{1}$ University of Lethbridge, Lethbridge, Alberta T1K 3M4, Canada, ${ }^{2}$ University of California, Irvine, California 92697, and ${ }^{3}$ Neuroelectronic Research \\ Flanders, 3000 Leuven, Belgium
}

The hippocampus is thought to contribute to episodic memory by creating, storing, and reactivating patterns that are unique to each experience, including different experiences that happen at the same location. Hippocampus can combine spatial and contextual/episodic information using a dual coding scheme known as "global" and "rate" remapping. Global remapping selects which set of neurons can activate at a given location. Rate remapping readjusts the firing rates of this set depending on current experience, thus expressing experience-unique patterns at each location. But can the experience-unique component be retrieved spontaneously? Whereas reactivation of recent, spatially selective patterns in hippocampus is well established, it is never perfect, raising the issue of whether the experiential component might be absent. This question is key to the hypothesis that hippocampus can assist memory consolidation by reactivating and broadcasting experience-specific "index codes" to neocortex. In CA3, global remapping exhibits attractor-like dynamics, whereas rate remapping apparently does not, leading to the hypothesis that only the former can be retrieved associatively and casting doubt on the general consolidation hypothesis. Therefore, we studied whether the rate component is reactivated spontaneously during sleep. We conducted neural ensemble recordings from CA3 while rats ran on a circular track in different directions (in different sessions) and while they slept. It was shown previously that the two directions of running result in strong rate remapping. During sleep, the most recent rate distribution was reactivated preferentially. Therefore, CA3 can retrieve patterns spontaneously that are unique to both the location and the content of recent experience.

Key words: CA3; episodic memory; hippocampus; memory reactivation; rate remapping

\section{Significance Statement}

The hippocampus is required for memory of events and their spatial contexts. The primary correlate of hippocampal activity is location in space, but multiple memories can occur in the same location. To be useful for distinguishing these memories, the hippocampus must be able, not only to express, but also to retrieve both spatial and nonspatial information about events. Whether it can retrieve nonspatial information has been challenged recently. We exposed rats to two different experiences (running in different directions) in the same locations and showed that even the nonspatial components of hippocampal cell firing are reactivated spontaneously during sleep, supporting the conclusion that both types of information about a recent experience can be retrieved.

\section{Introduction}

The hippocampus plays a central role in episodic memory. A prevailing theory behind this idea is that the CA3 region is a general-purpose auto-associative network (Marr, 1971; McNaughton and Morris, 1987; Treves, 1990; Treves and Rolls,

\footnotetext{
Received April 30, 2015; revised May 27, 2016; accepted June 2, 2016

Author contributions: C.D.S. and B.L.M. designed research; C.D.S. performed research; C.D.S., Z.N., K.A., and M.T. analyzed data; C.D.S., Z.N., and B.L.M. wrote the paper.

This work was supported by Alberta Innovates Health Solutions (Polaris Award to B.L.M. and Graduate Fellowship to C.D.S.), NSERC (Discovery Grants 418422-2012 and 429438-2012 to B.L.M. and Grant DG 386522-2010 to M.T.), and Neuroelectronic Research Flanders (B.L.M.).

The authors declare no competing financial interests.

Correspondence should be addressed to Dr. Bruce McNaughton, University of Lethbridge, 4401 University Drive West, Lethbridge, Alberta T1K3M4, Canada. E-mail: bruce.mcnaughton@uleth.ca.

DOI:10.1523/JNEUROSCI.1678-15.2016

Copyright $\odot 2016$ the authors $\quad 0270-6474 / 16 / 369342-09 \$ 15.00 / 0$
}

1992) capable of representing external inputs and encoding them in an attractor framework (Hopfield, 1982; Amit and Treves, 1989) using Hebbian synaptic modification. This allows the whole of the hippocampal representation of an event to be recalled from a reduced or distorted cue ("pattern completion"). It is generally believed that the spontaneous reactivation during sleep (Wilson and McNaughton, 1994) of patterns stored autoassociatively in the hippocampus serves to assist neocortical memory consolidation (Marr, 1971).

The hippocampus combines spatial and "contextual" information by means of a dual coding scheme in which the location and spatial extent of "place fields" appears to be determined by an internally generated mechanism based on path integration (McNaughton et al., 2006) signals, probably of medial entorhinal origin (Hafting et al., 2005; Fyhn et al., 2007), whereas the intensity of "in-field" firing is adjusted rapidly on the basis of sensory 
and internal cues ("context"), probably conveyed via lateral entorhinal cortex (Hargreaves et al., 2005; Lu et al., 2013). Rearrangement of the population of place field locations with respect to one another due to changing position is referred to as "global remapping," whereas cue-induced variations of in-field firing rates is called "rate remapping" (Leutgeb et al., 2005). Rate remapping has been observed in various conditions, such as changing wall color (Leutgeb et al., 2005), wall shape (Leutgeb et al., 2005; Leutgeb and Moser, 2007; Colgin et al., 2010; Lu et al., 2013), task demands (Allen et al., 2012), odors (Anderson and Jeffery, 2003), and internal variables such as working memory and/or action plans (Wood et al., 2000; Bower et al., 2005). In a recent study (Navratilova et al., 2012), we showed that the firing rate differences that occur when a rat runs in opposite directions on the same track (McNaughton et al., 1983) are a result of rate remapping that takes place in the first few laps.

Colgin et al. (2010) tested whether both spatial and sensory cue information can lead to attractor dynamics in CA3. They showed that, when the cues were gradually morphed between two familiar end points, coherent, abrupt transitions occurred in CA3 only if the end points had been learned previously in different spatial locations and thus were associated with different global mappings. They concluded that "associations among the represented features of the two boxes were not sufficient to produce attractor dynamics in the hippocampus, whereas associations between the features and an internal representation of relative location produced strong attractor dynamics," and thus, "the available evidence does not favor the theory that CA3 is a standard auto-associative network whose purpose is to encode associations among the sensory features of the environment." A major implication of this conclusion is that spontaneous retrieval of recently experienced patterns might contain only the spatial component of the activity and any component due to rate remapping would be lost. This result would present difficulties for most theories of the role of hippocampus in encoding episodic memories that occur at the same place.

We therefore tested the hypothesis that both the spatial and the rate-remapping components of firing (i.e., the full hippocampal code for the episode) are reactivated during sleep. We took advantage of the pronounced rate remapping that develops as a consequence of experience during running in opposite directions around a circular track (Navratilova et al., 2012). Essentially, we investigated whether the reactivation of the firing rate distribution that occurs during rest immediately after running in one direction is more similar to the hippocampal activation for that direction than for the pattern during subsequent running in the opposite direction. Rejection of the null hypothesis (no difference) in the predicted direction would indicate that both the spatial and the sensory features are retrieved in the hippocampus during offline reactivation.

\section{Materials and Methods}

Behavioral training and recording experiment. The animals used for this study were individually housed and kept on a $12 \mathrm{~h}$ dark $/ 12 \mathrm{~h}$ light cycle. The training and experiment were conducted during the light phase. The animals were food deprived and kept at $85 \%$ of their free feeding body weight to be motivated to run for food reward. All animal protocols complied with Federation of Laboratory Animal Science Associations guidelines under the guidance of the Neuro-Electronic Research Flanders and KU Leuven institutional animal care.

Four male Long-Evans and 1 male Wistar rat between 3 and 5 months of age were pretrained to run back and forth on a linear track for a food reward (chocolate sprinkles), which was provided on each end of the track. Training took place once or twice daily for approximately $2-3$ weeks depending on how quickly the animal acquired the task. During this training period, a "hyperdrive" with 12 independently movable recording tetrodes (Gothard et al., 1996) $(<1 \mathrm{M} \Omega)$ and two reference electrodes was implanted above the right hippocampus $(-3.8 \mathrm{AP},-3.0 \mathrm{ML}$ relative to bregma). The tetrodes were lowered into the cortex $(1960 \mu \mathrm{m})$ right after surgery and then further down to area CA3 of the hippocampus over the course of $\sim 2$ weeks. One tetrode was placed into layer CA1 to record ripple events. A reference tetrode was placed close to the hippocampal fissure to record sharp wave events and was used as a visual guide during tetrode turning. A differential signal was recorded from the corpus callosum and was used as neutral reference throughout the recordings. The tetrode turning was based on hippocampal local field potential (LFP) landmark features such as theta rhythm during waking and movement and the occurrence of sharp waves and ripples during quiet wakefulness and slow-wave sleep (Buzsáki et al., 1992). The anatomical positions of the tetrodes were confirmed histologically using Nissl stains (Fig. 1B).

Once all tetrodes were in the desired location, the experiment started. The recordings were conducted in a different room than the one used for pretraining with a circular track elevated $\sim 15 \mathrm{~cm}$ above the floor with low $(1 \mathrm{~cm})$ railings along the inside and outside (diameter: $170 \mathrm{~cm}$ ). The room and experimental apparatus were novel on day one of recording.

The recording procedure for $4 \mathrm{~d}$ ( 3 animals) to $5 \mathrm{~d}$ ( 2 animals) was conducted as follows (Fig. 1A). The animal first rested in a highwalled, towel-padded black wooden box placed in the center of the track for $\sim 45 \mathrm{~min}$. It then ran the first session of continuous laps in one direction for food reward at a fixed location on the track. The animal ran a total of four sessions lasting $15 \mathrm{~min}$ with the first and last sessions in the same direction $\left(\mathrm{A}, \mathrm{A}^{\prime}\right)$ and the second and third sessions $\left(\mathrm{B}, \mathrm{B}^{\prime}\right)$ in the same direction but opposite to $\mathrm{A}$. The running directions were switched each day such that $\mathrm{A}$ and $\mathrm{A}^{\prime}$ refer to clockwise (CW) running on days 1,3 , and 5 , and counterclockwise (CCW) running on days 2 and 4 . That is, each rat ran CW during the first and last sessions and CCW during the middle sessions on days 1, 3 and 5 and CCW-CW-CW-CCW on days 2 and 4. The reward location remained the same for each animal. Between the run sessions, the animal rested for $\sim 20 \mathrm{~min}$ in the box and, after the fourth session, it rested for $45 \mathrm{~min}$. The recording arena was surrounded by black curtains that were not fully closed to allow the experimenter to go in and out between sessions. The gap in the curtains at the door together with an illuminated emergency exit light above the door also provided a constant distant cue for the animal to polarize the environment. In addition, the experimenter sat on a chair close to the food dish to deliver the reward. The light was dimmed during recordings.

The thresholded spike signals were recorded with a Cheetah Data Acquisition System (Neuralynx), digitized at $32 \mathrm{kHz}$, and band-pass filtered between $600 \mathrm{~Hz}$ and $6 \mathrm{kHz}$. The continuous LFP traces were sampled at $2.4 \mathrm{kHz}$ and filtered between $1 \mathrm{~Hz}$ and $300 \mathrm{~Hz}$. The animal's position was tracked at 60 frames/s using LEDs mounted on the head stage that were detected by a color camera that was mounted on the ceiling of the recording room $(\sim 0.33 \mathrm{~cm} /$ pixel $)$.

Spike sorting. Spiking activity was analyzed offline using an automated spike-sorting algorithm (Klustakwik by K.D. Harris) to isolate units and to separate them from noise. The resulting clusters were manually refined using cluster cutting software (MClust 3.1SE by A.D. Redish, with customizations by P. Lipa, S. Cowen, and D. Euston) in a multiparameter space including features such as energy (area under the waveform), peak-to-trough distance, principal component, time (to control for stability of the recording of the unit over the entire length of recording), and cross-correlograms. Only units with $<0.2 \%$ interspike intervals falling within the 2 ms refractory period were accepted. In addition, the median of the $z$-scored waveforms of the last sleep session had to be within 1 SD of the median of the $z$-scored waveforms of the first sleep session on at least 3 of 4 channels to exclude units that underwent drift. By these criteria, we found 1058 units in the 22 datasets. Units that fired with $<0.05 \mathrm{~Hz}$ in all of the behavioral ( 494 cells) or all of the sleep ( 3 cells) sessions were excluded from the analysis (except for the cross-correlation analysis 

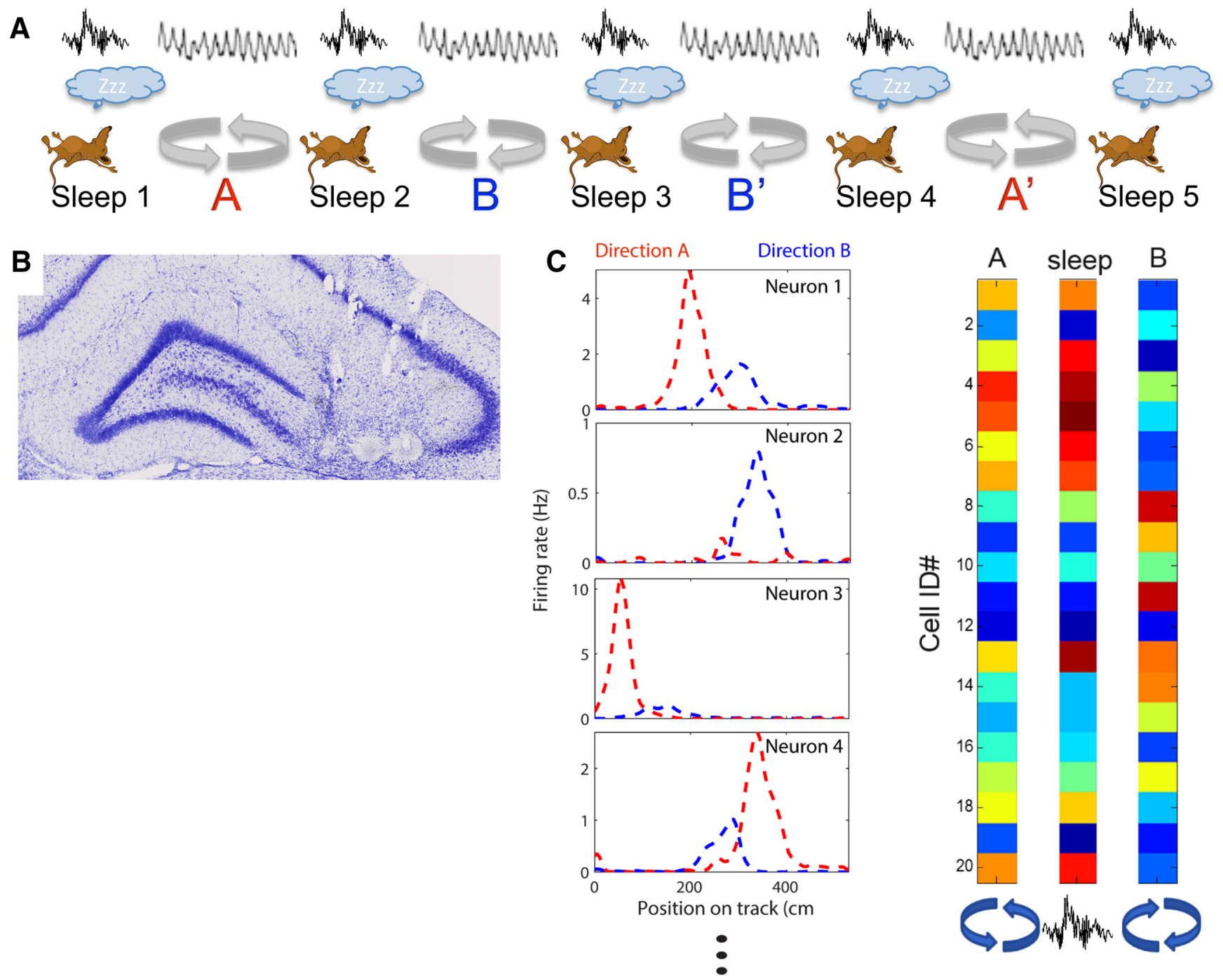

Neuron 20

Figure 1. Experimental methods. $A$, Behavioral sequence of the experiment. Animals rested before and after each of four running sessions. In the first and last running sessions, the animals ran in one direction in a circular track to receive a food reward at one location (sessions $A$ and $A^{\prime}$ ). In the other two running sessions, the rats ran along the same track, but in the opposite direction to receive a reward at the same location (sessions B and B'). During the rest periods, sharp-wave ripple complexes were recorded in CA1 and CA3. B, Representative histological section. A coronal slice through the dorsal hippocampus of rat 9 shows 3 lesions in $C A 3$, which were made at the electrode tips after all recording sessions were completed. Tracks of additional tetrodes are also visible above the CA3. C, Analysis procedure. The average firing rates of each cell during each running and rest session were calculated and compared. The critical comparison is between running session $A$ and the sleep immediately following (Sleep 2) and the same sleep with running session B, which had not yet been experienced that day.

between CA3 unit activity and ripple peak times), which left a total of 561 neurons for firing rate correlation analyses.

Place field analysis. The video-tracked position of the rat during the run session was extracted at each video frame by fitting a circle to the ring of LEDs on the head stage. The 2D position was deconstructed into a $1 \mathrm{D}$ position along the track. The food dish location was defined as 0 . The rat's velocity was calculated by smoothing the $2 \mathrm{D}$ position with a $1 \mathrm{~s}$ hamming window and calculating the distance the animal had moved between subsequent frames. Any periods in which the animal moved $<2 \mathrm{~cm} / \mathrm{s}$ were removed. Place fields were defined along the linearized track by smoothing (with a hanning window of 7 bins) the firing rate in $5 \mathrm{~cm}$ position bins for each cell. The smoothed firing rates were $z$-scored to 0 mean with a SD of 1 . Any peaks exceeding a $z$-score of 2 for at least $6 \mathrm{~cm}$ were detected as fields. All adjacent bins on either side of the peak were included until the $z$-score dropped to 0 , which is where the boundaries of the place field were set. Fields that were $<20 \mathrm{~cm}$ apart were merged and fields with peaks within $30 \mathrm{~cm}$ before or after the food dish location were deleted. First, sessions of same direction were combined and then field boundaries were deter- mined in each direction independently. If the fields overlapped in the two directions, they were matched and considered as one field. If no opposite direction match was found, then the same boundaries were used for both directions. This procedure ensured that all spikes of the fields were considered even when fields showed a backward shift in the running direction. All fields detected by the algorithm were checked manually and deleted if they did not show one single cycle of phase precession in at least one of the two directions and a total of 277 fields remained. Approximate 3\% place field boundaries were adjusted manually to contain one full cycle of phase precession and no more.

Spatial correlations. The spatial correlations were computed by correlating the linearized spatial maps of a cell in sessions of same or different running directions with each other (Fig. 1C). For different running directions, sessions $\mathrm{A}$ and $\mathrm{B}$ and sessions $\mathrm{A}^{\prime}$ and $\mathrm{B}^{\prime}$ were compared.

Population vector correlations in the mazes. The similarity of the spatial maps for same and different running directions was estimated by computing the population vector correlations. The linearized spatial maps of all $n$ cells were used to construct an $n$ cell (rows) by $t$ spatial bin (col- 


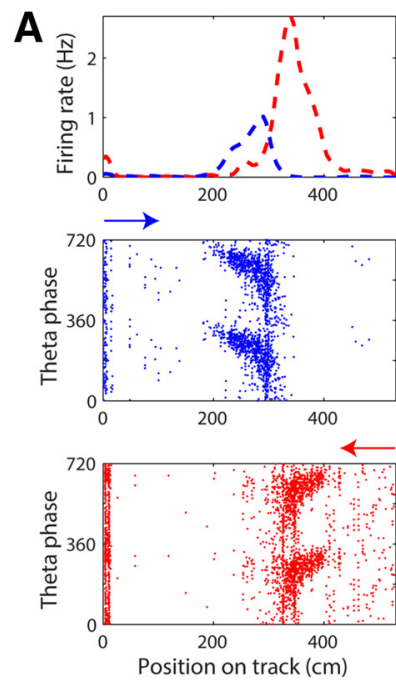

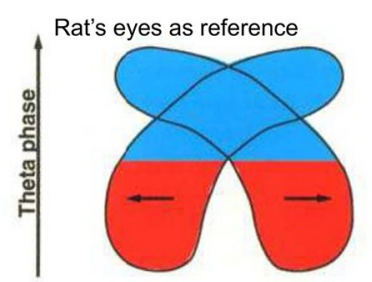
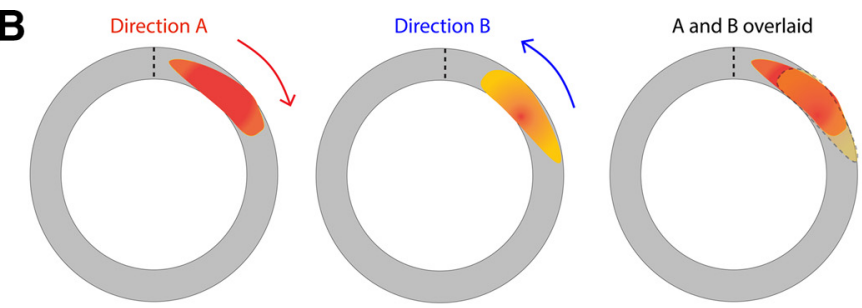

Back of the head as reference
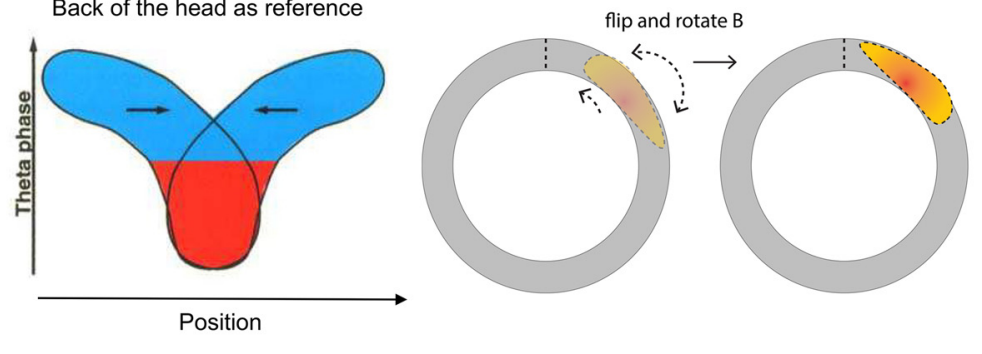

\section{All spatial maps were normalized by their maximum}

\section{C}

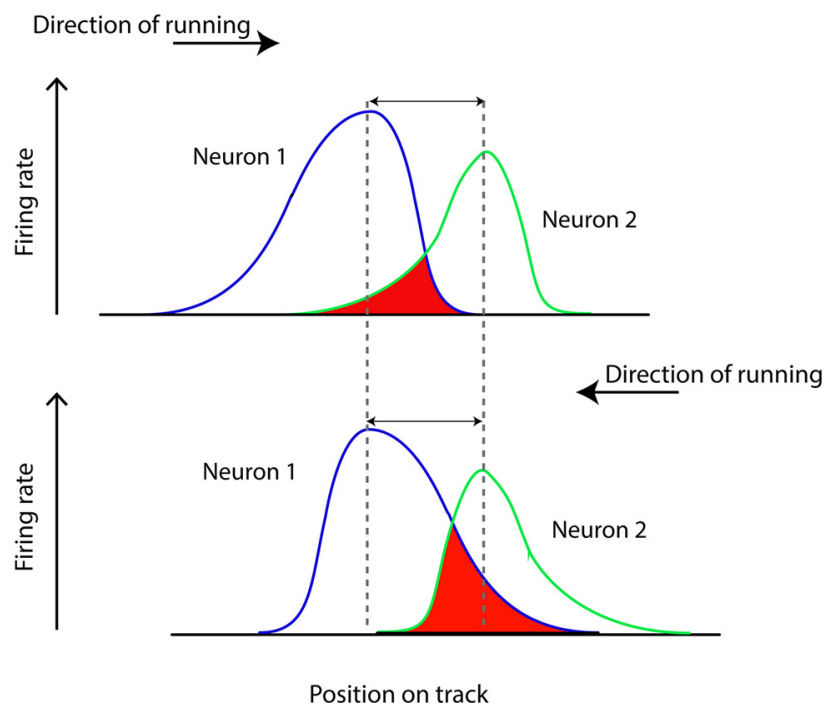

Figure 2. Asymmetry of place fields and its effect on the analysis. $A$, Place fields show an offset between running directions, depending on the reference position of the animal. The left panels show an example place field that has a field in both directions (session A and B), but shows a slight offset. The normalized firing rate against position on the track (top) and the theta phase of each spike plotted against position on the track are displayed. Direction $A$ and $B$ are marked blue and red and the arrows above the phase plots indicate the running direction. The right panels illustrate the effect of the reference position of the animal on the position of the spikes in various theta phases (modified from Skaggs et al., 1996). When the back of the head is taken as reference (as is the case in our recordings), only the spikes in the late theta phase will overlap (bottom). $\boldsymbol{B}$, Illustration of the manipulation of place fields for spatial correlation analyses. The positions of a field in direction $A$ and $B$ on the circular track are displayed first. Firing rate is false color coded, with warmer colors indicating higher rates. The right-most schematic illustrates that, when simply overlaying the sessions in different running directions, the field does not line up due to both the field offset shown in part A and field asymmetry. Therefore, the field in direction B is first flipped and then rotated by an amount calculated for each dataset (bottom left) to maximize the overlap of the place field in the two directions (bottom right). $C$, Hypothetical asymmetric place fields of two neurons (blue and green) are drawn on the track ( $x$-axis). The fields show expansion opposite to the animal's running direction (black arrows). The two cells show different place field locations, but each shows the same peak firing location and rate ( $y$-axis) in the two directions. The Euclidean distance (double sided arrow) between their peak firing locations (dashed line) is constant in the two directions. The area in which the firing of the two place cells overlaps is indicated in red and differs in the two directions. This difference will affect the correlation coefficient between the neuron pair in the two directions and bias the result toward finding a difference between the two directions and rejecting the null hypothesis when it is true (the individual neurons do not show rate remapping). Therefore, we only used net firing rate (over the whole track) for correlation analyses, which avoids this issue.

umns) matrix of firing rates for each condition (A, $\mathrm{A}^{\prime}$, i.e., first and last session and $\mathrm{B}, \mathrm{B}^{\prime}$, i.e., second and third session). For the different direction comparison, the two sessions of same running directions were combined ( $A$ and $\mathrm{A}^{\prime}$, as well as $\mathrm{B}$ and $\mathrm{B}^{\prime}$ ) and the rows in the spatial matrices were normalized by their maximum. The bins within the field boundaries in $\mathrm{B}$ (combined with $\mathrm{B}^{\prime}$ ) were flipped and shifted (Fig. $2 A, B$ ). The amount by which the fields were shifted was determined by what shift resulted in the highest mean spatial correlation over all cells per dataset. All cells within one dataset were shifted by the same amount. The population vector correlations were computed for each position bin in one spatial matrix with all position bins in another spatial matrix.

Global remapping simulation. Global remapping was simulated by moving the place fields of one direction randomly (independently for each cell) around the track and calculating the spatial correlations. This procedure was repeated 1000 times.

Directionality index (DI). The DI was computed by subtracting the number of spikes fired on each lap in the lower firing rate direction from the number of spikes fired in the higher firing rate direction within each field and dividing by the sum of the spikes fired on that lap in the two directions. The higher and lower firing rate directions were determined for each field by comparing the total firing rates during the entire sessions. This ensures that the running direction that is subtracted does not switch between laps (Navratilova et al., 2012). The DI can range between 0 and 1 , with 0 indicating an equal number of spikes in the 2 directions of comparison and 1 indicating zero firing in one direction but not the other. 

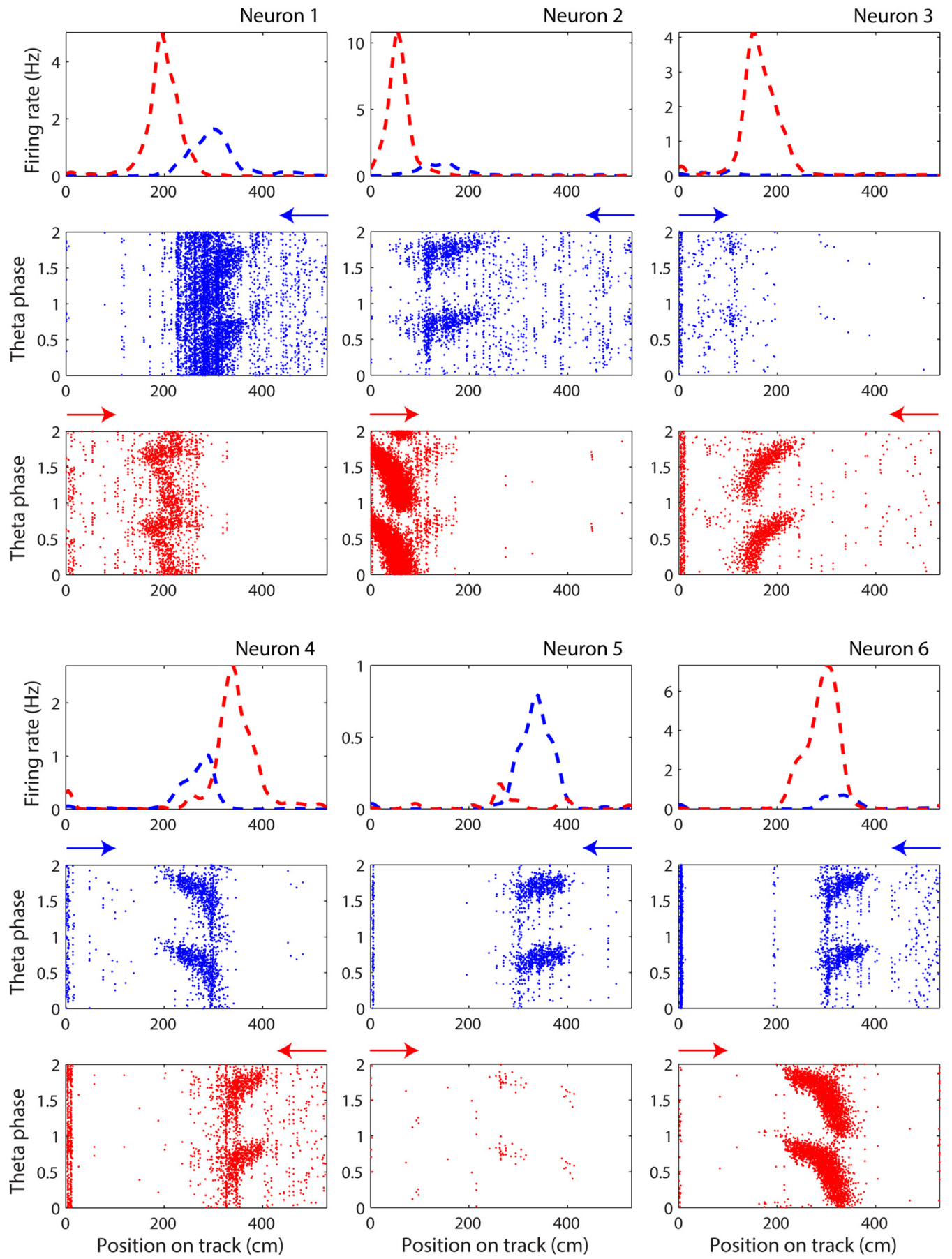

Figure 3. Rate remapping in different running directions on circular track. Six examples taken from 2 different rats on 3 different days illustrate the rate differences within place fields in different directions (indicated by colors blue and red). For each neuron, the normalized (i.e., occupancy corrected) firing rate is plotted in the two directions (blue and red dashed lines in first row) against the linearized position in centimeters on the track. The reward location is at $0 \mathrm{~cm}$. The theta phases of the spikes discharged in the two directions are plotted below. Two full cycles of theta phase are plotted for illustration purposes. Arrows over theta phase plots indicate running direction. Theta phase plots are not occupancy corrected, which is why there is an accumulation of spikes around the reward zone at the beginning of the track.

EEG analysis. The LFP signal recorded in the hippocampal fissure was filtered offline between 6 and $10 \mathrm{~Hz}$ to determine the theta signal for theta phase precession plots. To identify ripple events, the LFP recorded in the cell layer of CA1 was band-pass filtered offline between 100 and $300 \mathrm{~Hz}$ and the envelope of the signal was determined by a Hilbert transform and smoothed using a $30 \mathrm{~ms}$ sliding time window. Periods when the rat was motionless (i.e., moving $<2 \mathrm{~cm} / \mathrm{s}$ for at least $1 \mathrm{~s}$ ) were determined from video-tracking data for each sleep session and these periods were used to calculate average ripple power and detect ripples. Ripples were detected when the signal power continuously exceeded the mean plus $\sim 2 \mathrm{SD}$ for at least $15 \mathrm{~ms}$. Ripples were detected on all tetrodes and the tetrode on which ripples accounted for the highest proportion of spikes was used in further analysis. This was most often the tetrode in CA1, but sometimes it was one of the CA3 tetrodes. Ripple thresholds were adjusted manually to find a value that accounted for $\sim 16 \%$ of spikes fired during motionless periods. Each ripple had to reach a minimal peak power $(2-2.75$ SD over the mean) and the edges of the ripple were determined by finding the time points when the ripple dropped below a power threshold (1.5-2 SD over the mean) and expanding it by $25 \mathrm{~ms}$ on each edge. This method was found to capture the full increase in spike density surrounding ripples. 
A

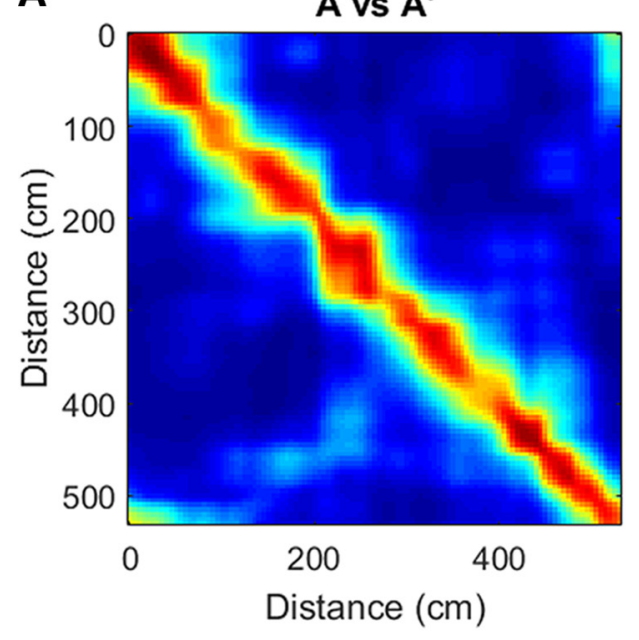

C

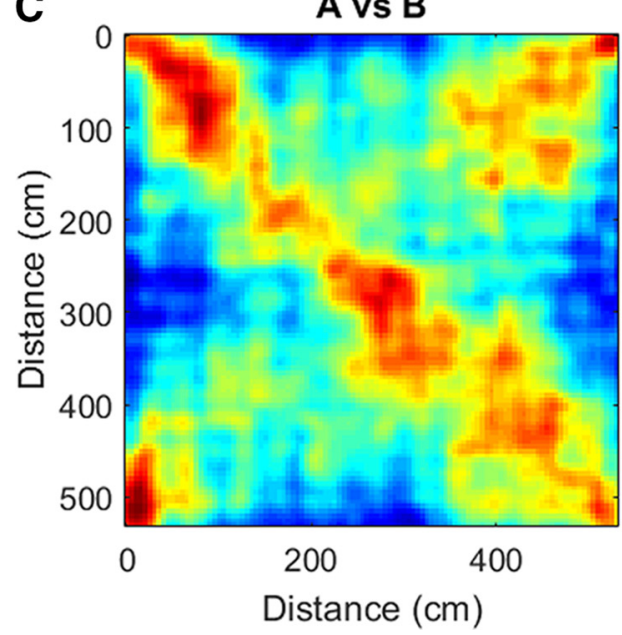

B

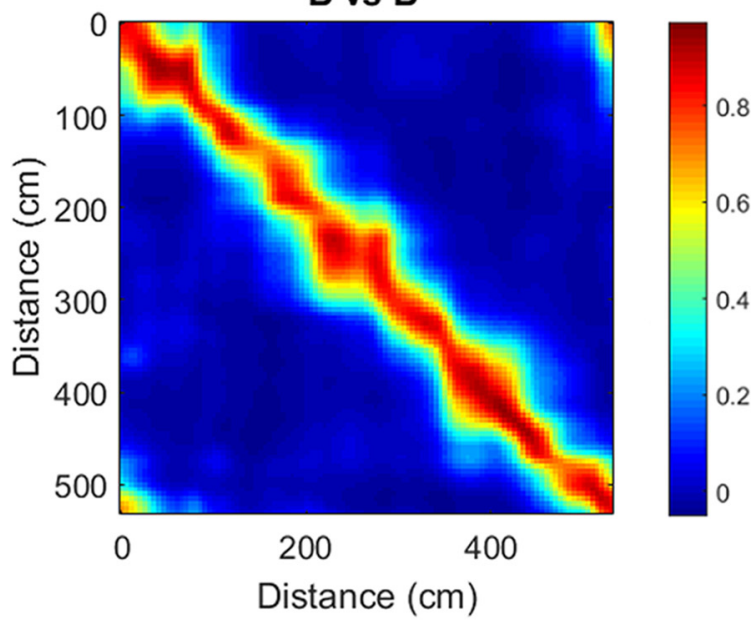

D Global remapping simulation

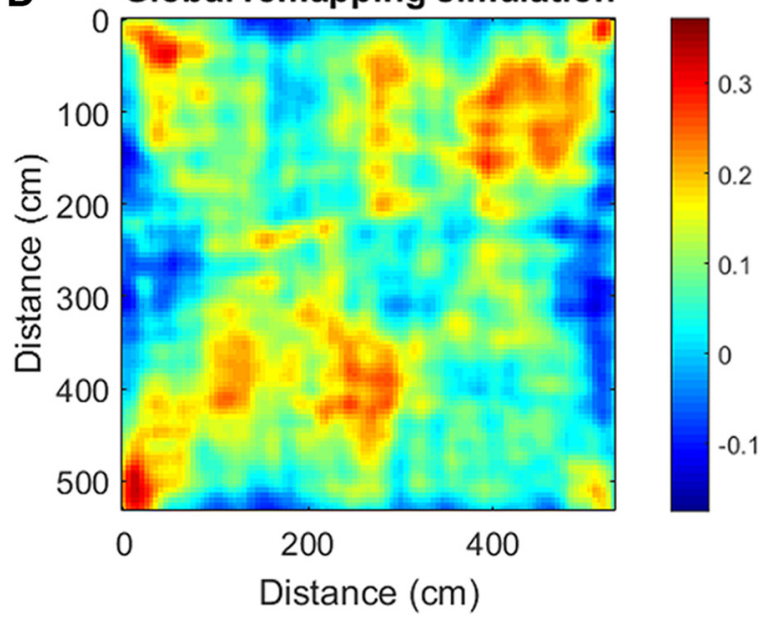

Figure 4. Similarity between spatial maps. $A$, The population vector correlations of the binned spatial maps (bin size: $5 \mathrm{~cm}$ ) of all neurons of the pooled data of all rats in session $A$ and $A^{\prime}$ (repeated recording in the same running direction; $\boldsymbol{A})$ and $B$ and $B^{\prime}(\boldsymbol{B})$ are plotted. Population vector correlations of corresponding position on the track (plotted along diagonal) show the highest values, whereas the values decrease with increasing offset between population vector locations. C, Population vector correlation matrix for sessions in opposite directions after normalizing each row to their maximum, flipping spatial bins within field boundaries and alignment of fields, shows lower values along the diagonal as well as a broader band. This effect arises from differences in firing rates as caused by rate remapping, asymmetric field expansion, and phase precession effects, which result in place fields in opposite directions being offset slightly when the rat's head is taken as the position reference (Fig. 14E in Skaggs et al., 1996). However, the spatial structure of field location in the two directions is preserved. $D$, Contrast with the rate remapping simulation, in which the spatial bins of all cells are randomly rotated around the track for one running direction. In this case, there is no correlation along the diagonal. $N=356$ cells with a maximal firing rate (in position bins) $>0.05$ $\mathrm{Hz}$ in any one running session. Top color bar applies to $\boldsymbol{A}$ and $\boldsymbol{B}$ and bottom color bar applies to $\boldsymbol{C}$ and $\boldsymbol{D}$.

Rate reactivation analysis. The mean firing rates of all neurons on each recording day were computed for each maze session during running periods (speed of rat $>2 \mathrm{~cm} / \mathrm{s}$ ) and for each sleep session during ripple periods. Because the firing rate distribution is lognormal (see Fig. $6 \mathrm{~A}$ ), the mean rates were log transformed. The correlations between the logtransformed firing rate vectors during sleep 2 were computed with the preceding (A) and following (B) maze sessions.

Cross-correlation analysis between CA3 unit activity and ripple peak times. Spike times of all CA3 neurons $(n=1058)$ were crosscorrelated with the ripple peak times recorded on that day as reference events. The correlograms were computed with a bin size of $5 \mathrm{~ms}$ over a window of $400 \mathrm{~ms}$ centered around 0 . The mean and SEM per bin were computed.

Pairwise correlations. A classical approach to assessing reactivation is to compute the pairwise correlation coefficients between neurons during sleep and behavior (Wilson and McNaughton, 1994; Kudrimoti et al., 1999). In our case, the critical comparisons would be the correlation coefficients for neuron pairs in maze A and for neuron pairs in S2 (same for maze B and S2). However, this approach is biased toward rejecting the null hypothesis due to the asymmetry of place fields, which can result in differences in the overlap of neighboring fields in opposite running directions, even in the absence of rate remapping (Fig. $2 C$ ). We computed the analysis and obtained significant results consistent with our conclusion; however, given the potential bias, we decided to not rely on this approach.

\section{Results}

To test whether hippocampal CA3 neurons reactivate the sensory/ contextual modulation of their rates within a stable spatial map, we recorded from five rats (561 neurons) while they were running on a circular track for 15 min per session in CW and CCW directions in an $\mathrm{A}-\mathrm{B}-\mathrm{B}^{\prime}-\mathrm{A}^{\prime}$ paradigm $\left(\mathrm{A}^{\prime}\right.$ and $\mathrm{B}^{\prime}$ are repeated recordings in the $\mathrm{A}$ and $\mathrm{B}$ directions). The experimental design is illustrated in Figure 1. Sleep epochs were recorded with the animal resting in a box in the center of the track (but not on the track) between run sessions for 20 $\min$ (S2-S4). Sleep sessions before the first (S1) and after the last (S5) running session were $45 \mathrm{~min}$ long. 

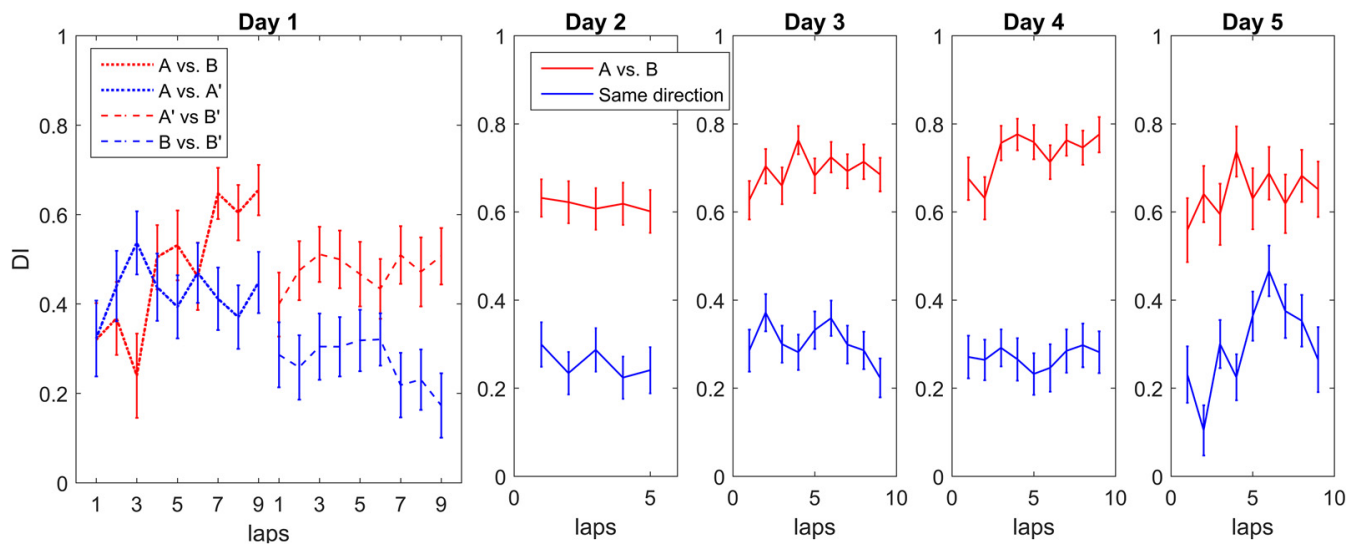

Figure 5. Rate remapping is measured with the DI. The DI, defined as the difference in the number of spikes fired per lap in the two directions divided by the sum of the number of spikes of a neuron, is plotted for each recording day and pooled over animals. The mean and SEM of the DI in the different direction comparison (red, $A$ vs $B$ and $A^{\prime}$ vs $B^{\prime}$ ) and same direction comparison (blue, $A$ vs $A^{\prime}$ and $B$ vs $B^{\prime}$ ) over all animals for each lap are plotted (Wilcoxon rank-sum test, one-tailed, day $1, p=0.004$, days $2-5, p<0.0001$ ). For day 1 , the Dls in the first two sessions ( $A$ vs $B$ ) were not significantly different from the A versus $A^{\prime}$ comparison, but the DI increased over laps, as shown previously (Navratilova et al., 2012 ). By sessions 3 and 4 ( $A^{\prime}$ vs $B^{\prime}$ ), the Dls were significantly different from the same direction comparison. On Days $2-5$, the Dls in the first 2 sessions did not differ from the Dls in the last 2 sessions and thus were plotted together for clarity. Laps are cut off at the worst-performing animal on that day.

\section{Firing rate encodes running direction within a stable spatial code}

CA3 neurons with place fields on the circular track show directiondependent firing rate differences (Fig. 3). The selected examples in Figure 3 show phase precession in their fields in both directions, but the absolute firing rates are direction specific. The location is largely constant except for an asymmetry due to phase precession effects (see below). These results reproduce the findings from Navratilova et al. (2012) showing that direction-dependent firing rate changes are not indicative of global remapping because many cells fire and show phase precession in both running directions even though the firing rates may be quite different. For a quantitative assessment of whether the spatial maps for the two running directions are not different in terms of spatial location of the place fields of each cell (i.e., no global remapping; Fig. $4 A-C$ ), we assessed the similarity using population vector correlations.

For same direction comparisons (Fig. $4 A, B$ ), the population vector correlations show a narrow band with values close to 1 along the diagonal. This indicates that field locations and within-field firing rates are similar in repeated recordings in the same direction. Correlation values further away from the diagonal correspond to positions farther apart on the track and therefore appear more and more dissimilar. For different direction comparisons (Fig. 4C), all rows in the binned spatial maps of all cells were normalized by their maximum to alleviate the influence of rate difference and to emphasize the similarity of the two spatial maps. The bins within field boundaries in one direction were flipped and rotated by an optimal amount assessed per dataset to compensate for the field asymmetry (place fields in opposite directions are slightly offset when the rat's head is taken as the position of reference due to phase precession effects and asymmetrical place field expansion; Skaggs et al., 1996; Fig. 2A). This improved the cell-by-cell spatial correlations significantly $(0.11 \pm$ 0.14 compared with $0.24 \pm 0.20$ after flipping and rotating, $p=$ $0.0007, t$ test, one-tailed, $n=22$ datasets; 256 cells with place fields included from all datasets). Both rotated and nonrotated spatial correlations are greater than a simulated global remapping situation (Fig. $4 D$ shows a random remapping simulation), in which the fields in one direction were rotated randomly along the track (each cell's fields rotated a different random amount, repeated 1000 times: $-0.0014 \pm 0.098$, compared with shifted bins, $t$ test, one-tailed: $p<$ 0.000001 , compared with nonshifted bins, $t$ test, one-tailed: $p=$
0.0010). The 95th percentile global remapping simulations were calculated for each dataset $(0.16 \pm 0.088)$ and, in 15 of 22 datasets, the A versus B correlation was $>95 \%$ of the global remapping simulations. The highest correlation values are lower than in the same direction comparisons and form a wider band along the diagonal (average cell-by-cell spatial correlation in same direction comparison: $0.60 \pm 0.14)$. The increased width of the band is due to the fact that different cells have differently sized place fields, so the offset in fields between running directions is slightly different for each cell, whereas we adjusted the offset uniformly for all cells in the dataset.

\section{Reactivation in CA3 includes contextual modulation of firing rates}

Next, we quantified the effect of rate remapping using a DI (Leutgeb et al., 2005; Navratilova et al., 2012) that measures the similarity of firing of a cell in two conditions. The DI is the absolute difference of the number of spikes of a cell fired per lap over the sum of the spikes and ranges between 0 (i.e., there is an equal number of spikes in both laps compared and there is no sampling error) and 1 (i.e., the number of spikes fired in one direction is zero and the other is nonzero). We calculated the DI by comparing the number of spikes fired within the place field boundaries in opposite directions (Fig. 5, red) with the number of spikes within the place field boundaries in the same directions (Fig. 5, blue). The DI for opposite directions is significantly different from the DI for same directions already on the second half of the first day (Wilcoxon rank-sum test, one-tailed, $n=56$ fields, $p=0.21$, A vs $\mathrm{B}$ compared with $\mathrm{A}$ vs $\mathrm{A}^{\prime}, p=0.0025, \mathrm{~A}^{\prime}$ vs $\mathrm{B}^{\prime}$ compared with $\mathrm{B}$ vs $\left.B^{\prime}\right)$. Therefore, the rate remapping was partly established on the first day (Navratilova et al., 2012) and increased on the subsequent recording days (Wilcoxon rank-sum test, one-tailed, $p<$ 0.00001 for days $2-5$, all sessions for opposite vs same direction).

To determine whether the firing rates are faithfully reactivated in sleep after behavior, we tested the following null hypothesis. If CA3 only reactivates the spatial information provided by path integration input, which is identical in both sessions, we expect that activity in session A explains activity in the following sleep (S2) no better than activity in the subsequent run session B [order: A - sleep (S2) - B]. The specific alternative hypothesis is that both spatial and rate-remapped components are reactivated and therefore activity (i.e., firing rates) in A explains activity in sub- 
A

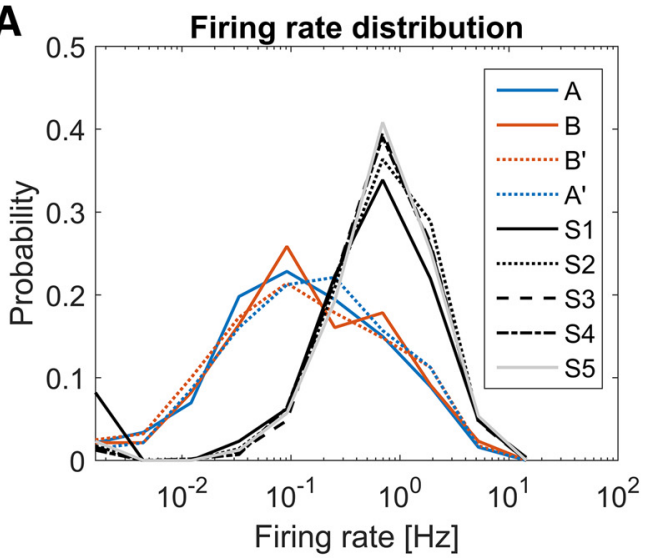

C Average correlation coefficient

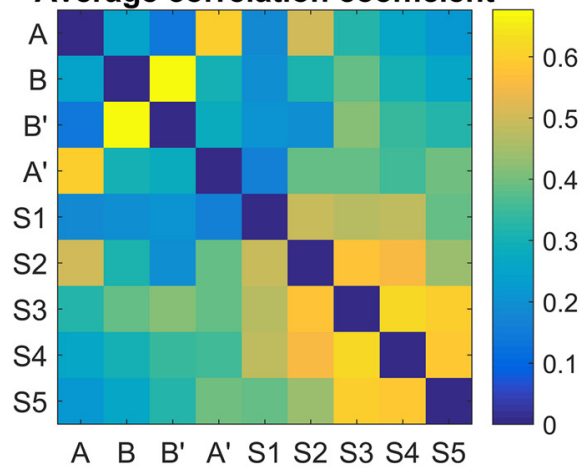

B

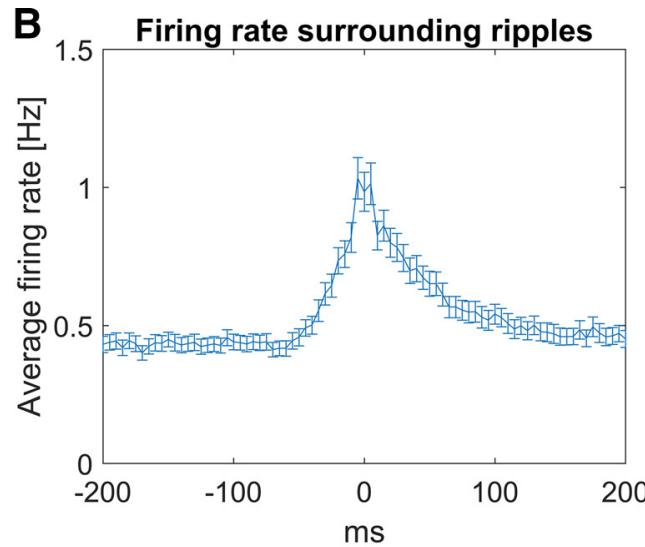

D

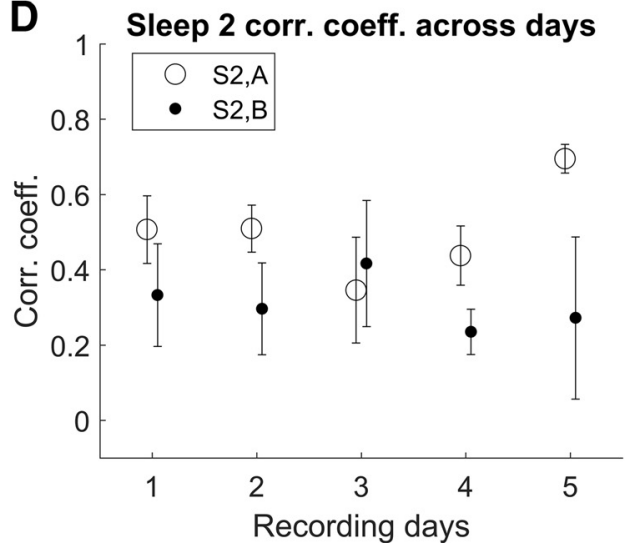

Figure 6. Lognormal firing rate distribution, $\mathrm{CA} 3$ unit activity with respect to ripple peak times and the reactivation of rate remapping. $A$, Firing rate distribution of all recorded single units is plotted for all behavioral (red and blue solid and dashed lines) and all sleep sessions during ripples (black and gray solid, dashed and dotted dashed lines). Note the log scale on the rate axis: the firing rate distribution of CA3 neurons is lognormal in each session. $B$, CA3 cell activity cross-correlation with ripple peak times in the EEG with a bin size of $5 \mathrm{~ms}$. The mean and SEM are plotted for each bin. CA3 neurons show elevated firing within a window of $-30 \mathrm{~ms}$ to $+70 \mathrm{~ms}$ around the peak of ripple oscillations recorded in the CA1 pyramidal layer. $C$, Mean firing rates of all cells in all running and rest sessions were log transformed. Then the population vector for each rat and each day was correlated between all sessions. The correlation matrix represents the average correlation over all recording sessions $(n=22)$. Correlations of each session with itself are by definition 1 (along the unity line), but these values are removed for a clearer comparison between sessions. Note that behavior sessions $A$ vs $A^{\prime}$ and $B$ vs $B^{\prime}$ show the highest correlations, as do correlations between subsequent sleep sessions. Among the running versus sleep session correlations (upper right and lower left quadrants), sleep 2 versus $A$ stands out as the most correlated. $D$, Correlation between $\mathrm{S} 2$ and $\mathrm{A}$ (open circles), as well as between $\mathrm{S} 2$ and B (closed circles), are plotted over the 5 recording days. Error bars represent the SEM calculated across rats (day 1-4: $n=5 ;$ day $5: n=2$ ). The correlations between $\mathrm{S} 2$ and $\mathrm{A}$ are significantly higher than between $\mathrm{S} 2$ and $\mathrm{B}$ (paired $t$ test, one tailed, $n=22$ recording sessions, $p=0.028$ ).

sequent sleep better than activity in $\mathrm{B}$ does. Because the firing rate distributions in behavior and sleep are lognormal (Fig. 6A), we computed the correlations between the log-transformed mean firing rate vectors (Battaglia et al., 2005; Mizuseki and Buzsáki, 2013) during running periods in sessions (A and B) and during ripple periods in sleep S2 (Fig. 6C). The correlation $r(\mathrm{~S} 2, \mathrm{~A})$ was significantly higher than $r(\mathrm{~S} 2, \mathrm{~B})[r(\mathrm{~S} 2, \mathrm{~A})=0.47 \pm 0.21, r(\mathrm{~S} 2$, $\mathrm{B})=0.32 \pm 0.27$, Student's $t$ test, one-tailed, $n=22$ datasets, $p=$ 0.028 ] over the course of 5 recording days. We can therefore reject the null hypothesis that firing rates in sleep 2 are determined purely by the spatial component and not the nonspatial component of the prior experience.

\section{Discussion}

CA3 has been shown to encode contextual information flexibly onto a single spatial map without affecting other independent spatial maps (Gothard et al., 1996; Anderson and Jeffery, 2003; Leutgeb et al., 2005, 2007; Allen et al., 2012). In addition, it has been demonstrated that CA3 reactivates pattern sequences in sleep after the experience, but lack of perfect fidelity in this reactivation and the apparent lack of attractor dynamics for the sensory components during waking left open the question of whether reactivation during sleep contains both sensory and spa- tial components or only the latter. We found that firing rates in sleep were significantly more similar to the previously experienced episode than to a future episode of identical spatial content with different sensory context, confirming that both determinants of the collective activity are reactivated.

Global remapping reflects a situation in which a population of neurons represents changes in an environment as an all-or-none phenomenon: changes in a variable (usually location in space, or perceived location in space) cause the whole population to change their response. This reflects discrete point attractor dynamics: small changes in the variable do not shift the representation, but changes beyond a specific threshold cause a large shift in the representation, which is the essence of pattern completion and pattern separation. In contrast, rate remapping reflects a situation in which the population of neurons can encode a variable (e.g., sensory components of the environment or behavioral goals) continuously so that small changes in the variable create small changes in the firing rates of the active neurons, but do not change the active population entirely. It has been shown previously that both types of dynamics exist in CA3 (Colgin et al., 2010). In this study, we have provided evidence that CA3 firing rates within sharp waves after an experience do reflect the sensory 
experience of the environment, showing that discrete attractor dynamics may not be necessary for replay.

Replay, like cued recall, is thought to be triggered from activity in a subset of the neurons involved in the initial memory formation (perhaps from random activity during slow-wave sleep), which causes pattern completion and activation of the full neural ensemble. If nonspatial content of the memory is stored in the firing rates of the neurons without discrete attractor dynamics, how can this content be recalled from a noisy or partial trace? Recent studies showed that the population output of CA3 showed abrupt attractor-like transitions when two initially spatially independent environments were morphed from one to the other (Leutgeb et al., 2005, 2007; Colgin et al., 2010), but when visually distinct environments are experienced from the start at the same location, morphing the sensory input causes diverse and noncoherent changes in CA3 neuron firing (Leutgeb et al., 2005, 2007; Colgin et al., 2010; Lu et al., 2013). Some cells do show abrupt transitions, but do so at different points, and some cells show smooth transitions and others show hysteresis (the firing rates are affected by recent experiences, not just the current one); however, as a population, the transition in the output appears gradual (Colgin et al., 2010). This suggests that there might not be discrete attractor dynamics for sensory/episodic information in CA3. Recent modeling studies, however, suggest that episodic memory could be supported by a combination of discrete and continuous attractor dynamics (Rennó-Costa et al., 2014; Solstad et al., 2014). The possibility of discrete and continuous attractor dynamics in the recurrent collaterals of CA3 and also hysteresis effects in some cells suggests that different sensory/episodic events that occur in the context of the same spatial map may be stored in the synapses of CA3 neurons and be amenable to retrieval during offline reactivation.

\section{Conclusion}

This study shows that CA3 combines and stores spatial and episodic components of memories. CA3 is able to retrieve specific episodic information related to a single spatial location spontaneously. It remains to be investigated whether two or more episodic memories for the same spatial location can coexist in the same CA3 network. If that is the case, then rate remapping may be an effective way of encoding and separating different experiences associated with the same location and using this separation to index the different memory components in the neocortex. We attempted to find evidence of replays of multiple episodes at the same location in our data, but due to the low number of active CA3 cells per location (sparsity), it was not possible to reach a definitive conclusion on this question.

\section{References}

Allen K, Rawlins JN, Bannerman DM, Csicsvari J (2012) Hippocampal place cells can encode multiple trial-dependent features through rate remapping. J Neurosci 32:14752-14766. CrossRef Medline

Amit DJ, Treves A (1989) Associative memory neural network with low temporal spiking rates. Proc Natl Acad Sci U S A 86:7871-7875. CrossRef Medline

Anderson MI, Jeffery KJ (2003) Heterogeneous modulation of place cell firing by changes in context. J Neurosci 23:8827-8835. Medline

Battaglia FP, Sutherland GR, Cowen SL, Mc Naughton BL, Harris KD (2005) Firing rate modulation: a simple statistical view of memory trace reactivation. Neural Netw 18:1280-1291. CrossRef Medline

Bower MR, Euston DR, McNaughton BL (2005) Sequential-contextdependent hippocampal activity is not necessary to learn sequences with repeated elements. J Neurosci 25:1313-1323. CrossRef Medline
Buzsáki G, Horváth Z, Urioste R, Hetke J, Wise K (1992) High-frequency network oscillation in the hippocampus. Science 256:1025-1027. CrossRef Medline

Colgin LL, Leutgeb S, Jezek K, Leutgeb JK, Moser EI, McNaughton BL, Moser MB (2010) Attractor-map versus auto-association based attractor dynamics in the hippocampal network. J Neurophysiol 104:35-50. CrossRef Medline

Fyhn M, Hafting T, Treves A, Moser MB, Moser EI (2007) Hippocampal remapping and grid realignment in entorhinal cortex. Nature 446: 190-194. CrossRef Medline

Gothard KM, Skaggs WE, McNaughton BL (1996) Dynamics of mismatch correction in the hippocampal ensemble code for space: interaction between path integration and environmental cues. J Neurosci 16:80278040. Medline

Hafting T, Fyhn M, Molden S, Moser MB, Moser EI (2005) Microstructure of a spatial map in the entorhinal cortex. Nature 436:801-806. CrossRef Medline

Hargreaves EL, Rao G, Lee I, Knierim JJ (2005) Major dissociation between medial and lateral entorhinal input to dorsal hippocampus. Science 308: 1792-1794. CrossRef Medline

Hopfield JJ (1982) Neural networks and physical systems with emergent collective computational abilities. Proc Natl Acad Sci U S A 79:25542558. CrossRef Medline

Kudrimoti HS, Barnes CA, McNaughton BL (1999) Reactivation of hippocampal cell assemblies: effects of behavioral state, experience, and EEG dynamics. J Neurosci 19:4090-4101. Medline

Leutgeb JK, Moser EI (2007) Enigmas of the dentate gyrus. Neuron 55: 176-178. CrossRef Medline

Leutgeb JK, Leutgeb S, Moser MB, Moser EI (2007) Pattern separation in the dentate gyrus and CA3 of the hippocampus. Science 315:961-966. CrossRef Medline

Leutgeb S, Leutgeb JK, Barnes CA, Moser EI, McNaughton BL, Moser MB (2005) Independent codes for spatial and episodic memory in hippocampal neuronal ensembles. Science 309:619-623. CrossRef Medline

Lu L, Leutgeb JK, Tsao A, Henriksen EJ, Leutgeb S, Barnes CA, Witter MP, Moser MB, Moser EI (2013) Impaired hippocampal rate coding after lesions of the lateral entorhinal cortex. Nat Neurosci 16:1085-1093. CrossRef Medline

Marr D (1971) Simple memory: a theory for archicortex. Philos Trans R Soc Lond B Biol Sci 262:23-81. CrossRef Medline

McNaughton BL, Morris RGM (1987) Hippocampal synaptic enhancement and information-storage within a distributed memory system. Trends Neurosci 10:408-415. CrossRef

McNaughton BL, Barnes CA, O'Keefe J (1983) The contributions of position, direction, and velocity to single unit activity in the hippocampus of freely-moving rats. Exp Brain Res 52:41-49. Medline

McNaughton BL, Battaglia FP, Jensen O, Moser EI, Moser MB (2006) Path integration and the neural basis of the 'cognitive map'. Nat Rev Neurosci 7:663-678. Medline

Mizuseki K, Buzsáki G (2013) Preconfigured, skewed distribution of firing rates in the hippocampus and entorhinal cortex. Cell Rep 4:1010-1021. CrossRef Medline

Navratilova Z, Hoang LT, Schwindel CD, Tatsuno M, McNaughton BL (2012) Experience-dependent firing rate remapping generates directional selectivity in hippocampal place cells. Front Neural Circuits 6:6. CrossRef Medline

Rennó-Costa C, Lisman JE, Verschure PF (2014) A signature of attractor dynamics in the CA3 region of the hippocampus. PLoS Comput Biol 10:e1003641. CrossRef Medline

Skaggs WE, McNaughton BL, Wilson MA, Barnes CA (1996) Theta phase precession in hippocampal neuronal populations and the compression of temporal sequences. Hippocampus 6:149-172. CrossRef Medline

Solstad T, Yousif HN, Sejnowski TJ (2014) Place Cell Rate Remapping by CA3 Recurrent Collaterals. PLoS Comput Biol 10:e1003648. CrossRef Medline

Treves A (1990) Threshold-linear formal neurons in auto-associative nets. Journal of Physics A: Mathematical and General 23:2631-2650. CrossRef

Treves A, Rolls ET (1992) Computational constraints suggest the need for two distinct input systems to the hippocampal CA3 network. Hippocampus 2:189-199. CrossRef Medline

Wilson MA, McNaughton BL (1994) Reactivation of hippocampal ensemble memories during sleep. Science 265:676-679. CrossRef Medline

Wood ER, Dudchenko PA, Robitsek RJ, Eichenbaum H (2000) Hippocampal neurons encode information about different types of memory episodes occurring in the same location. Neuron 27:623-633. CrossRef Medline 